Regards sur l'économie allemande

Bulletin économique du CIRAC

$113 \mid 2014$

Varia

\title{
Emploi : réduire le mismatch des emplois peu qualifiés
}

\section{Isabelle Bourgeois}

\section{(2) OpenEdition \\ 1 Journals}

Édition électronique

URL : http://journals.openedition.org/rea/4700

DOI : $10.4000 /$ rea. 4700

ISSN : 1965-0787

Éditeur

CIRAC

\section{Édition imprimée}

Date de publication : 15 juillet 2014

Pagination : 45-46

ISSN : 1156-8992

\section{Référence électronique}

Isabelle Bourgeois, "Emploi : réduire le mismatch des emplois peu qualifiés », Regards sur l'économie

allemande [En ligne], 113 | juillet 2014, mis en ligne le 17 juillet 2016, consulté le 15 septembre 2020

URL : http://journals.openedition.org/rea/4700

Ce document a été généré automatiquement le 15 septembre 2020.

(c) CIRAC 


\title{
Emploi : réduire le mismatch des emplois peu qualifiés
}

\author{
Isabelle Bourgeois
}

1 Une grande part du chômage en Allemagne (le taux de chômage aux critères OIT est actuellement de 5,1 \%) s'explique par le manque de qualification des actifs. Et près de la moitié des demandeurs d'emploi $(45 \%)$ doit de ce fait se contenter d'une activité d'auxiliaire. De surcroit, les perspectives de trouver un tel emploi faiblement qualifié varient énormément selon les régions : elles sont particulièrement faibles dans les villes de la Ruhr (Gelsenkirchen, Herne, Duisburg, Recklinghausen Dortmund et Oberhausen), où le taux de chômage dans cette catégorie atteint $40 \%$, ainsi que dans l'est de l'Allemagne ( $35 \%)$. Formulé autrement: plus l'économie régionale est compétitive, plus elle crée d'emplois y compris peu qualifiés.

\section{Quelle politique pour l'emploi ciblant les moins qualifiés?}

2 Ce sont là les principales conclusions d'une étude publiée le 16 juin par l'Institut IAB près de l'Agence fédérale pour l'emploi de Nuremberg (IAB-Kurzbericht, 11/2014). Dans une approche visant à rendre plus efficiente la politique active de l'emploi, elle analyse minutieusement la relation entre niveau des qualifications et type d'emploi occupé ou recherché. Comme, au fil de ses mutations vers une "économie des savoirs et de la connaissance » et au fil de la hausse générale des qualifications, l'emploi non qualifié a massivement reculé, il s'agit de définir une politique permettant de mieux intégrer à l'avenir ceux qui ne disposent pas d'un diplôme sanctionnant une formation professionnelle achevée (voir dans ce numéro l'étude de R. Lasserre sur la formation professionnelle) ou dont la formation ne correspond plus aux exigences du marché du travail moderne. 


\section{Réduire le mismatch par une hausse des qualifications}

3 Il s'agit donc de réduire le mismatch entre offre et demande dans le segment des faibles qualifications. Car l'offre d'emplois faiblement qualifiés existe bel et bien. L'IAB a ainsi décelé un stock de 4 millions d'emplois disponibles dans des fonctions auxiliaires, notamment dans l'entretien, la sécurité, la logistique, l'hôtellerie/gastronomie, l'agriculture, la santé et les services sociaux. Mais ce réservoir est très inégalement réparti sur le territoire. Et la difficulté pour accéder à ces emplois dans certaines régions reflète la structure économique de celles-ci. Ainsi, dans celles où l'agriculture a un poids important, l'accès est relativement facile; il l'est encore plus dans celles marquées par une forte activité industrielle qui requiert l'ensemble de la gamme des qualifications et des savoir-faire. Mais face à une demande globalement bien supérieure à l'offre d'emplois dans ce segment, l'appel à la mobilité de la main-d'œuvre n'est pas une solution adaptée. La priorité de la politique active pour l'emploi doit résider au contraire dans un effort de qualification visant à réduire progressivement le nombre d'actifs peu ou pas qualifiés s'inscrivant au chômage.

\section{Une nouvelle lecture des emplois et un critère clé : les compétences individuelles}

Pour réduire ce mismatch (qui concerne, rappelons-le, une grande partie des bénéficiaires du régime d'assistance Hartz IV), il faut en avoir une lecture plus fine. C'est ainsi que l'IAB recourt à une nouvelle classification des métiers (Klassifikation der Berufe, KldB) développée en 2010 par l'Agence fédérale pour l'emploi pour accroître la flexibilité dans sa politique pour l'emploi et sa gestion du placement. Cette classification dresse une sorte de bilan global ou holistique des compétences, indépendamment des formations suivies ou acquises; elle tient compte de la complexité des tâches requises dans chaque métier, donc de l'évolutivité des compétences et aptitudes individuelles. Les emplois (ou métiers) se trouvent ainsi classés en 4 catégories correspondant, au fond, à un niveau d'exigence en termes de savoir-faire : (1) auxiliaire, (2) personne qualifiée, (3) spécialiste et (4) expert.

5 En règle générale, plus la formation initiale est élevée, plus l'emploi occupé se rapproche du haut de cette hiérarchie : ainsi, en juin 2013, 86 \% des actifs de 25 à 64 ans occupaient un emploi soumis à cotisations sociales exigeant au moins un diplôme d'ouvrier professionnel. Or l'étude de l'IAB se concentre sur tous les autres, ceux du bas de l'échelle, classés dans la catégorie "auxiliaire » (Helfer). Celle-ci répond au niveau d'exigence suivant: activités peu complexes, travail de routine; pas de formation professionnelle (sous aucune forme). Autrement dit, cette catégorisation vise à prendre en considération les compétences (au sens d'employabilité) de ceux dont la qualification ne peut pas être attestée par un diplôme.

\section{Un tiers des actifs d'origine étrangère sont « auxiliaires »}

Cette catégorie se compose pour une grande partie d'actifs d'origine étrangère : alors que $13 \%$ seulement (ouest; $12 \%$ à l'est) des Allemands de souche entrent dans cette 
catégorie, les migrants sont $34 \%$ à l'ouest et $30 \%$ à l'est. L'IAB voit dans cette forte proportion d'étrangers parmi les "auxiliaires » l'effet de la politique d'immigration menée par la RFA (ouest) dans les années 1960, dont l'objectif était de recruter massivement une main-d'œuvre non qualifiée ; à l'inverse, les Länder de l'Est mènent aujourd'hui une politique d'immigration sélective, ce qui explique les 4 points de différence entre l'est et l'ouest de l'Allemagne. De même, on constate que les "auxiliaires" sont souvent plus âgés; la raison réside dans l'évolution des qualifications requises sur le marché du travail au cours des dernières décennies. Quant aux femmes, elles sont $16 \%$ (ouest) à occuper une fonction/emploi « d'auxiliaire »; les hommes ne sont que $13 \%$.

Classification par niveau d'exigence des actifs occupant un emploi soumis à cotisations sociales (en \%)

\begin{tabular}{|c|c|c|c|c|}
\hline & " auxiliaire " & $\begin{array}{l}\text { "personne } \\
\text { qualifiée " }\end{array}$ & « spécialiste" & « expert » \\
\hline $\begin{array}{l}\text { Total (3,657 millions d'actifs } \\
\text { occupés) }\end{array}$ & 14 & 58 & 14 & 14 \\
\hline \multicolumn{5}{|l|}{ Par sexe et par région } \\
\hline Hommes, ouest & 13 & 55 & 16 & 16 \\
\hline Femmes, ouest & 16 & 62 & 11 & 11 \\
\hline Hommes, est & 13 & 60 & 13 & 14 \\
\hline Femmes, est & 13 & 61 & 12 & 13 \\
\hline \multicolumn{5}{|l|}{ Par nationalité et par région } \\
\hline Allemands, ouest & 13 & 59 & 15 & 14 \\
\hline Etrangers, ouest & 34 & 49 & 7 & 10 \\
\hline Allemands, est & 12 & 61 & 13 & 14 \\
\hline Etrangers, est & 30 & 44 & 8 & 18 \\
\hline \multicolumn{5}{|l|}{ Par tranche d'âge } \\
\hline $25-34$ ans & 13 & 59 & 14 & 15 \\
\hline $35-44$ ans & 13 & 58 & 14 & 14 \\
\hline $45-54$ ans & 15 & 58 & 14 & 13 \\
\hline
\end{tabular}




\begin{tabular}{|l|l|l|l|l|}
\hline 55 ans et plus & 16 & 58 & 12 & 13 \\
\hline
\end{tabular}

NB : " auxiliaire » : tâches simples, travail de routine, aucune qualification professionnelle ; "personne qualifiée » : savoir et savoir-faire techniques requis, formation professionnelle achevée ; "spécialiste» : tâches complexes, fonctions d'encadrement, diplôme de Meister (contremaître/maître artisan) ou études supérieures équivalentes ; " expert » : tâches hautement complexes, fonctions de direction, au moins bac+4 ou expérience professionnelle équivalente. Etat : juillet 2013.

Source des données : D. Bogai, T. Buch, H. Seibert, « Arbeitsmarktchancen von Geringqualifizierten. Kaum eine Region bietet genügend einfache Jobs », IAB-Kurzbericht, 11/2014.

\section{Le diplôme n'est plus un critère d'employabilité}

Plus les qualifications des actifs sont faibles, plus le risque d'entrer ou de rester au chômage comme celui de rester au pied de l'ascenseur social sont donc élevés. La conclusion qu'en tirent les auteurs est que la réponse la mieux adaptée à ces personnes en difficulté réside d'une part dans une lutte ciblée contre l'échec scolaire ou durant la formation professionnelle, une orientation professionnelle plus adéquate encore, un effort plus systématique de formation continue et, bien sûr, une meilleure reconnaissance des diplômes étrangers. D'autre part, en matière de placement, une plus grande flexibilité est requise: il convient en effet "d'évaluer au cas par cas si, lorsqu'il n'y a pas de concordance avec le niveau d'exigence d'un emploi, les compétences et capacités individuelles du demandeur d'emploi ne sont pas de nature à justifier son placement malgré tout ». La qualification - et surtout l'employabilité - d'un demandeur d'emploi ne se résument pas au diplôme...

\section{INDEX}

Mots-clés : emploi, politique de l'emploi, emploi peu qualifié, qualification, compétence, auxiliaire, diplôme 